\title{
Three-dimensional Analysis of the Distance between Pulp Tissue and Caries or Obturation in Different Projections using CBCT
}

\section{Federico Valenti Obino, Dario Di Nardo, Linda Quero*, Gabriele Miccoli, Gianluca Gambarini, Luca Testarelli and Massimo Galli}

Department of Oral and Maxillo Facial Sciences, "Sapienza" University of Rome, Italy

*Corresponding Author: Linda Quero, Department of Oral and Maxillo Facial Sciences, "Sapienza" University of Rome, Italy.

Received: July 24, 2019; Published: August 26, 2019

DOI: $10.31080 /$ ASDS.2019.03.0623

\begin{abstract}
Aim: The aim of this research was to analyze the distance between radiographic pulp chamber and caries or obturations in vivo using cone-beam computed tomography (CBCT) imaging in different projections.

Materials and Methods: A total of 50 premolar and molar teeth from 50 patients were examined using CBCT imaging, previously taken for diagnosis and treatment. Slices in different projections were scanned and the distance between radiographic pulp chamber and caries or obturations was measured using a software's tool.

Results: The distance in two-dimensional projection was significantly lower than in three-dimensional projection in all the examined teeth, showing a less distance from the pulp chamber and the caries or obturation.

Conclusions: The distance between the caries and the pulp tissue of a tooth in a three-dimensional projection is less than in a twodimensional projection; for this reason, it would be better to pay attention to the proximity to the pulp chamber and it is recommended to consider a smaller distance than the one the two-dimensional radiograph showed.
\end{abstract}

Keywords: Pulp Tissue; CBCT; Distance

\section{Introduction}

An accurate diagnosis of caries is essential for clinicians, to have an exact knowledge of the depth of the cavity in order to determine the right type of restoration and treatment required [1].

Various studies have examined different methods, both intraoral and extraoral, to determine the extension of a caries, such as microcomputed tomography [2], swept-source optical coherence tomography [3], near-infrared transillumination [4], histological analysis [5], high-frequency ultrasound [6], bitewing radiography [7], periapical radiography [8], panoramic [9], conebeam computed tomography [10].
Extraoral radiography such as the panoramic had an inferior performance compared with intraoral imaging due to the superimposition of additional structures, increased image blurriness and inconsistent opening of posterior proximal contacts [11].

The intraoral radiography is widely used for diagnosis of proximal caries that cannot be readily identified by clinical examination [12].

CBCT imaging produces three dimensional images with high resolution and its radiation dose is lower than computed tomography [13]; the main disadvantage of the CBCT is the 
possibility of metal artefacts due to the presence of these materials within the oral cavity [14].

Our understanding of the caries disease process has identified lesion depth, activity and cavitation status as significant indicators for caries progression $[15,16]$.

Assessment of cavitation is also important because cavitated lesions demonstrate a much higher chances of progression $[17,18]$.

The aim of this research was to analyze the distance between the pulp chamber and the caries or obturations in premolars and molars using and comparing 3D radiographs: more precisely CBCT visualized with a sagittal projection (PS images) in a buccallingual sense (similar to the two-dimensional periapical endoral radiography) and CBCT visualized with a coronal projection (images in PC) in a mesio-distal sense (hidden anatomy).

\section{Materials and Methods}

Sample selection: A total of 50 premolar and molar teeth were examined. Teeth were selected from the CBCT examinations of 50 patients (27 males and 23 females) with an age ranging between 18 and 79 years. Images were obtained from CBCT examinations as part of diagnosis and treatment planning of patients who required large field of view for other reasons. The research was approved by the Ethical Committee of Policlinico Umberto I, Rome, Italy (ref. $582 / 17)$.

The samples were selected according to the following criteria

- $\quad$ Class II Black's Classification of caries;

- Deep caries that does not reach the pulp chamber.

All devitalized teeth were excluded.

The teeth were divided into two groups

- Distance between obturation and pulp chamber (figure 1);

- $\quad$ Distance between caries and pulp chamber (figure 2).

Image acquisition

CBCT images had been taken using the GXDP-500 system (Gendex Dental, Biberach, Germany), operating at $90 \mathrm{kVp}$ and 7 $\mathrm{mA}$, with an exposure time of $23 \mathrm{~s}$ and a voxel size of $0.2 \mathrm{~mm}^{3}$, with a field of view of $13 \times 9 \times 13 \mathrm{~cm}$, with an estimated dose of about 5 $\mathrm{mSv}$, allowing measurements to an accuracy of $0.2 \mathrm{~mm}$.
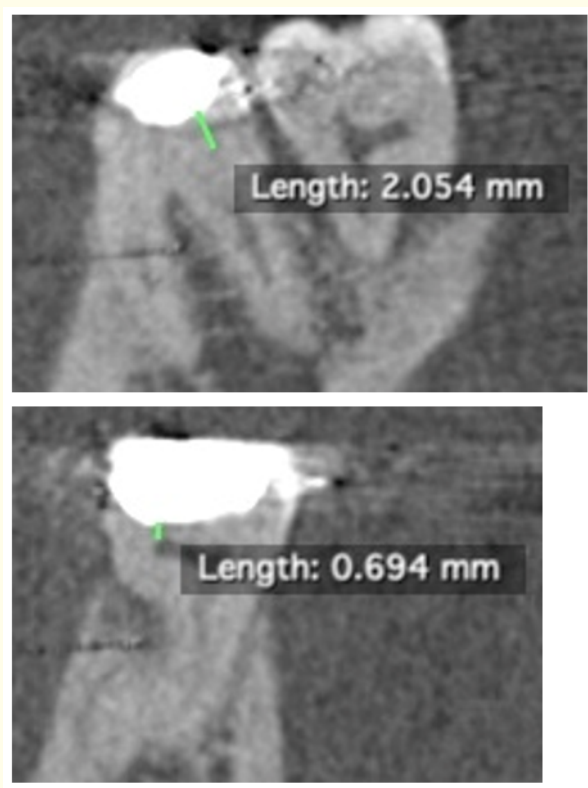

Figure 1: 2D vs 3D slice in obturations.
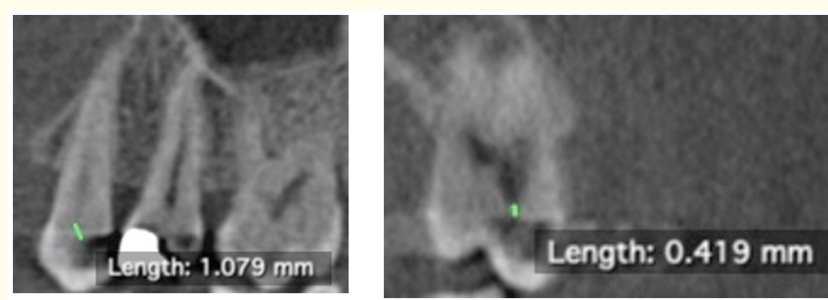

Figure 2: 2D vs 3D slice in caries.

Image evaluation

Through the use of Horos ${ }^{\mathrm{TM}}$ software (The Horos Project, 64-bit medical image viewer, GNU Lesser General Public Licence, version 3.0) three-dimensional reconstructions were analyzed to evaluate the parameters of interest. Images were reworked according to the axial, sagittal and coronal planes. CBCT images were viewed on reconstructions according to the axial plane, scrolling the cursor in the coronal-apical direction before, and then in the apical-coronal direction, to get a detailed view of caries and obturations in 2D and $3 \mathrm{D}$ slice. This action was repeated 3 times, and when the images in the axial plane were not clear, the tooth was also inspected in the three-dimensional reconstruction. The software had a specific tool which allowed precise measurements $(\sim 0,01 \mathrm{~mm})$. 


\section{Statistical analysis}

The results were analysed statistically using SPSS 20.0 (SPSS, Inc., Chicago, IL, USA) with the significance set at $\mathrm{p}<0.05$. One-way ANOVA was used for the association between the variables along with the post hoc tests, Tukey's HSD and Games-Howell. The t-test was used to compare the mean distances from confluence to radiographic root end.

\section{Results}

In the present study measurements made on PC images were significantly different from those performed on PS images.

In group A the average distance between the obturation and the pulp chamber is $2,157 \mathrm{~mm}$ in PS images and $1,403 \mathrm{~mm}$ in PC images. The difference measured about between the two projections was $0,754 \mathrm{~mm}$.

In group $B$ the average distance between the caries and the pulp chamber is $1,532 \mathrm{~mm}$ in PS images and $0,798 \mathrm{~mm}$ in PC images. The difference measured between the two projections was 0,734 $\mathrm{mm}$.

Overall, there was at least $0,7 \mathrm{~mm}$ of discrepance between PS and PC images, showing a more accurate capacity of PC projections in detecting the proximity of the pulp tissue both in caries and obturations.

\section{Discussion}

Defining the real extent of a caries has always been a challenge for the clinician, who must adopt different therapies based on the different severity of the process.

Standard bitewing radiographs detect only about $60 \%$ of proximal lesions [19-21].

Extraoral imaging had a significantly inferior performance compared with intraoral imaging due to superimposition of additional structures [22,23].

Many researches showed that CBCT caries detection results are approximately equivalent to intraoral modalities for non-restored teeth [24-30].

Other studies demonstrated that CBCT is equivalent to intraoral techniques at detecting clinically relevant caries lesions in minimally restored teeth; artefacts from metal objects and dense tooth structure (enamel) are limiting factors [3,32].

So, there was a minimal difference in detecting interproximal caries between 2D and 3D methods [33].

Previous researches that were performed on extracted teeth to define the accuracy of measurements of the caries using CBCT [3436] showed there was not statistical difference between 3D and 2D imaging in detecting interproximal caries.

Other studies [37-42] that compared the accuracy of detecting caries between 3D and 2D imaging demonstrated that the accuracy of CBCT may be similar to that of intraoral digital radiographic images for occlusal and proximal caries detection.

On the contrary, some researches [43-45] showed a significant superior ability of the CBCT to precisely define the extent of the carious process.

In particular, the use of CBCT overcomes the limitations of conventional radiography because it is a minimally invasive tool that can provide images displayed in coronal, sagittal and axial planes, a large number of teeth that can be examined with the same exposure to the X-rays, it can provide a precise location of the examined lesion with the possibility of its millimetrical measurement, it gives the possibility of making right and left symmetrical evaluations, it allows the study of the three-dimensionality of the tooth in its entirety. Moreover the most important feature of CBCT is that it reduce the superimposition of the surrounding structures [46].

The research performed by Takahashi., et al. [47] to assess the diagnostic property of intraoral bitewing radiographs (BTW) compared with periapical radiographs (PA) showed that there was no significant difference in the specificity of BTW and PAs; moreover, they founded that BTWs offer a significant advantage over PAs in the diagnoses of early stages of interproximal carious lesions.

Our study demonstrated that the distance between obturation and pulp chamber in group A in PC images was less than in PS images. The main distance in PS images was $2,157 \mathrm{~mm}(+/-0,2)$ and $1,403 \mathrm{~mm}(+/-0,2)$ in PC images. 
The distance between caries and pulp chamber in group B (table 2) also was less in PC images than in PS images. The main distance in PS images was $1,53 \mathrm{~mm}(+/-0,2)$ and $0,79 \mathrm{~mm}(+/-0,2)$ in PC images.

Overall, the distance concerning with caries and obturations (table 3) in PC images was significantly lower than PS mages. The main distance in PS images was $1,95 \mathrm{~mm}(+/-0,2)$ and $1,20 \mathrm{~mm}(+/-$ 0,2 ) in PC images.

Surely having a double vision from two different points of view means that in the PC images the distance between the lesion and the pulp chamber is shorter and therefore the caries is deeper.

Our results are totally new in literature, because in other studies it was showen that the diagnosis of caries depth was the same in both PS images and PC images [20-26].

A probable explanation for these results is the superposition of healthy structures: the distance in a mesio-vestibular direction between the caries and the pulp is smaller or the total thickness of dentin visible in PC images is smaller.

Our results underline the advantage of using CBCT; however, there is to understand if this advantage can be ensured with a compatible X-ray dose; it can be used a low dose protocol [48] or new machines [49].

In our study there was only one observer, unlike other researches; this can lead to a faster scan of the results, but on the other hand to a less precise evaluation of them [43].

Only in one study was measured the depth of the lesion [43], but only our study examined the millimetric distance between the lesion or the obturation and the pulp.

The clinical relevance is that this research can open up to various approaches of treatment of deep caries: selective caries removal [50], photodynamic therapy [51], antimicrobial composites [52], ozone application [53].

\section{Conclusions}

The average distance examined in PC images was lower in all cases compared to PS images both in the caries extension and in the obturations assessments.
This result demonstrates the lack of reliability of a twodimensional radiograph or of a single three-dimensional projection to examine the extent of a carious process and its presumable distance from the pulp tissue; this argument is superimposable also to evaluate the proximity of a previous obturation to the pulp tissue.

\section{Bibliography}

1. Park YS., et al. "Current status of dental caries diagnosis using cone beam computed tomography". Imaging Science in Dentistry 41.2 (2011): 43-51.

2. Zander V., et al. "Microcomputed Tomography Evaluation of Root Dentin Caries Prevention by Topical Fluorides and Potassium Iodide". Sensors (Bassel) 19.4 (2019): 874.

3. Suzuki S., et al. "Detection of enamel subsurface lesions by swept-source optical coherence tomography". Dental Materials - Journal 38.2 (2019): 303-310.

4. Lederer A., et al. "In vitro validation of near-infrared transillumination at $780 \mathrm{~nm}$ for the detection of carieson proximal surfaces". Clinical Oral Investigations (2019).

5. Berdouses ED., et al. "Histological validation of the automated caries detection system (ACDS) in classifying occlusal caries with the ICDAS II system in vitro. European Archives of Paediatric Dentistry (2018).

6. Kim J., et al. "High-Frequency Ultrasound Imaging for Examination of Early Dental Caries". Journal of Dental Research 98.3 (2019): 363-367.

7. Tonkaboni A., et al. "Comparison of diagnostic effects of infrared imaging and bitewing radiography in proximal caries of permanent teeth". Lasers in Medical Science (2018).

8. Akarslan ZZ., et al. "A comparison of the diagnostic accuracy of bitewing, periapical, unfiltered and filtered digital panoramic images for approximal caries detection in posterior teeth". Dentomaxillofacial Radiology 37.8 (2008): 458-463.

9. Akkaya N., et al. "Comparing the accuracy of panoramic and intraoral radiography in the diagnosis of proximal caries". Dentomaxillofacial Radiology 35.3 (2006): 170-174.

10. Qu X., et al. "Detection accuracy of in vitro approximal caries by cone beam computed tomography images". European Journal of Radiology 79.2 (2011): 24-27. 
11. Flint DJ., et al. "A diagnostic comparison of panoramic and intraoral radiographs". Oral Surgery, Oral Medicine, Oral Pathology, Oral Radiology, and Endodontology 85.6 (1998): 731-735.

12. Safi Y., et al. "Diagnostic accuracy of Cone Beam Computed Tomography, conventional and digital radiographs in detecting interproximal caries". Journal of Medicine and Life 8 (2015): 77-82.

13. Yildizer Keris E., et al. "Diagnostic accuracy of cone-beam computed tomography in detecting secondary caries under composite fillings: an in vitro study". Journal of Istanbul University Faculty of Dentistry 51.1 (2017): 22-27.

14. Nardi C., et al. "Metal and motion artifacts by cone beam computed tomography (CBCT) in dental and maxillofacial study". La radiologia medica 120.7 (2015): 618-626.

15. Baelum V. "What is an appropriate caries diagnosis?". Acta Odontologica Scandinavica 68.2 (2010): 65-79.

16. Kidd EA and Fejerskov O. "What constitutes dental caries? Histopathology of carious enamel and dentin related to the action of cariogenic biofilms". Journal of Dental Research 83 (2004): C35-8.

17. Sansare K., et al. "Clinical cavitation and radiographic lesion depth in proximal surfaces in an Indian population". Acta Odontologica Scandinavica 72.8 (2014): 1084-1088.

18. Ferreira Zandoná A., et al. "The natural history of dental caries lesions: a 4-year observational study". Journal of Dental Research 91.9 (2012): 841-846.

19. Dove SB. "Radiographic diagnosis of dental caries". Journal of Dental Education 65.10 (2001): 985-990.

20. Douglass CW., et al. "Clinical efficacy of dental radiography in the detection of dental caries and periodontal diseases". Oral Surgery, Oral Medicine, Oral Pathology, Oral Radiology, and Endodontology 62.3 (1986): 330-339.

21. Bader JD., et al. "Systematic reviews of selected dental caries diagnostic and management methods". Journal of Dental Education 6510 (2001): 960-968.

22. Flint DJ., et al. "A diagnostic comparison of panoramic and intraoral radiographs". Oral Surgery, Oral Medicine, Oral Pathology, Oral Radiology, and Endodontology 85.6 (1998): 731-735.
23. Akarslan ZZ., et al. "A comparison of the diagnostic accuracy of bitewing, periapical, unfiltered and filtered digital panoramic images for approximal caries detection in posterior teeth". Dentomaxillofacial Radiology 37.8 (2008): 458-463.

24. Park YS., et al. "Current status of dental caries diagnosis using cone beam computed tomography". Imaging Science in Dentistry 41.2 (2011): 43-51.

25. Rathore S., et al. "Ex vivo comparison of Galileos cone beam CT and intraoral radiographs in detecting occlusal caries". Dentomaxillofacial Radiology 41.6 (2012): 489-493.

26. Valizadeh S., et al. "Evaluation of cone beam computed tomography (CBCT) system: comparison with intraoral periapical radiography in proximal caries detection". Journal of Dental Research, Dental Clinics, Dental Prospects 6.1 (2012): 1-5.

27. Wenzel A., et al. "Detection of cavitated approximal surfaces using cone beam CT and intraoral receptors". Dentomaxillofacial Radiology 42.1 (2013): 39458105.

28. Tsuchida R., et al. "Evaluation of a limited cone-beam volumetric imaging system: comparison with film radiography in detecting incipient proximal caries". Oral Surgery, Oral Medicine, Oral Pathology, Oral Radiology, and Endodontology 104.3 (2007): 412-416.

29. Kalathingal SM., et al. "In vitro assessment of cone beam local computed tomography for proximal caries detection". Oral Surgery, Oral Medicine, Oral Pathology, Oral Radiology, and Endodontology 104.5 (2007): 699-704.

30. Kayipmaz S., et al. "An in vitro comparison of diagnostic abilities of conventional radiography, storage phosphor, and cone beam computed tomography to determine occlusal and approximal caries". European Journal of Radiology 80.2 (2011): 478-482.

31. Schulze R., et al. "Artefacts in CBCT: a review". Dentomaxillofacial Radiology 40.5 (2011): 265-273.

32. Draenert FG., et al. "Beam hardening artefacts occur in dental implant scans with the NewTom cone beam CT but not with the dental 4-row multidetector CT". Dentomaxillofacial Radiology 36.4 (2007): 198-203.

33. Gaalaas L., et al. "Ex vivo evaluation of new 2D and 3D dental radiographic technology for detecting caries". Dentomaxillofacial Radiology 45.3 (2016): 20150281. 
34. Kayipmaz S., et al. "An in vitro comparison of diagnostic abilities of conventional radiography, storage phosphor, and cone beam computed tomography to determine occlusal and approximal caries". European Journal of Radiology 80.2 (2011): $478-482$

35. Tsuchida R., et al. "Evaluation of a limited cone-beam volumetric imaging system: Comparison with film radiography in detecting incipient proximal caries". Oral Surgery, Oral Medicine, Oral Pathology, Oral Radiology, and Endodontology 104.3 (2007): 412-416.

36. Qu X., et al. "Detection accuracy of in vitro approximal caries by cone beam computed tomography images". European Journal of Radiology 79.2 (2011): 24-27.

37. Park YS., et al. "Current status of dental caries diagnosis using cone beam computed tomography". Imaging Science in Dentistry 41.2 (2011): 43-51.

38. Valizadeh S., et al. "Evaluation of cone beam computed tomography (CBCT) system: comparison with intraoral periapical radiography in proximal caries detection". Journal of Dental Research, Dental Clinics, Dental Prospects 6.1 (2012): 1-5.

39. Wenzel A., et al. "Detection of cavitated approximal surfaces using cone beam CT and intraoral receptors". Dentomaxillofacial Radiology 42.1 (2013): 39458105.

40. Tsuchida R., et al. "Evaluation of a limited cone- beam volumetric imaging system: comparison with film radiography in detecting incipient proximal caries". Oral Surgery, Oral Medicine, Oral Pathology, Oral Radiology, and Endodontology 104.3 (2007): 412-416.

41. Kalathingal SM., et al. "In vitro as- sessment of cone beam local computed tomography for proximal caries detection". Oral Surgery, Oral Medicine, Oral Pathology, Oral Radiology, and Endodontology 104.5 (2007): 699-704.

42. Kayipmaz S., et al. "An in vitro comparison of diagnostic abilities of conventional radiography, storage phosphor, and cone beam computed tomography to de- termine occlusal and approximal caries". European Journal of Radiology 80.2 (2011): 478-482.

43. Akdeniz BG., et al. "Accuracy of proximal caries depth measurements: Comparison between limited cone beam computed tomography, storage phosphor and film radiography". Caries Research 40.3 (2006): 202-207.
44. American Dental Association Council on Scientific A. "The use of cone-beam computed tomography in dentistry: An advisory statement from the american dental association council on scientific affairs". The Journal of the American Dental Association 143.8 (2012): 899-902.

45. Murat S., et al. "Visibility of artificial buccal recurrent caries under restorations using different radiographic techniques". Operative Dentistry 38.2 (2013): 197-207.

46. Gurtu A., et al. "CBCT: A revolutionary diagnostic aid for endodontic dilemmas". Minerva Stomatologica 63.9 (2014): 325331.

47. Takahashi N., et al. "A comparison of diagnosis of early stage interproximal caries with bitewing radiographs and periapical images using consensus reference". Dentomaxillofacial Radiology 48.2 (2019): 20170450.

48. Hidalgo Rivas JA., et al. "Development of a low-dose protocol for cone beam CT examinations of the anterior maxilla in children". The British Journal of Radiology 88.1054 (2015): 20150559.

49. Deman P., et al. "Dose measurements for dental cone-beam CT: a comparison with MSCT and panoramic imaging". Physics in Medicine and Biology 59.12 (2014): 3201-3222.

50. Bjørndal LB., et al. "Management of Deep Caries and the Exposed Pulp". International Endodontic Journal 52.7 (2019): 949-973.

51. Martins Reis AC., et al. "Scientific Evidence In Antimicrobial Photodynamic Therapy: An Alternative Approach For Reducing Cariogenic Bacteria". Photodiagnosis and Photodynamic Therapy (2019).

52. Karched M., et al. "In vivo antimicrobial activity of silver diammine fluoride on carious lesions in dentin". Journal of Oral Science 61.1 (2019): 19-24.

53. Durmus N., et al. "Effectiveness of the ozone application in two-visit indirect pulp therapy of permanent molars with deep carious lesion: a randomized clinical trial". Clinical Oral Investigations (2019).

\section{Volume 3 Issue 9 September 2019 (C) All rights are reserved by Linda Quero., et al.}

